\title{
A Note on Toric Varieties Associated to Moduli Spaces
}

\author{
James J. Uren
}

\begin{abstract}
In this note we give a brief review of the construction of a toric variety $\mathcal{V}$ coming from a genus $g \geq 2$ Riemann surface $\Sigma^{g}$ equipped with a trinion, or pair of pants, decomposition. This was outlined by J. Hurtubise and L. C. Jeffrey in [3]. In [6] A. Tyurin used this construction on a certain collection of trinion decomposed surfaces to produce a variety $D M_{g}$ - the so-called Delzant model of moduli space - for each genus $g$. We conclude this note with some basic facts about the moment polytopes of the varieties $\mathcal{V}$. In particular, we show that the varieties $D M_{g}$ constructed by Tyurin, and claimed to be smooth, are in fact singular for $g \geq 3$.
\end{abstract}

MSC Primary/Secondary: 14M25/52B20

\section{Introduction}

\section{$1.1 \quad$ Setting}

Let $\Sigma^{g}$ be a compact oriented two manifold of genus $g$, and suppose that $\partial \Sigma^{g}=\emptyset$. Let $\mathcal{M}\left(\Sigma^{g}\right)$ be the moduli space of gauge equivalence classes of flat $S U(2)$ connections on $\Sigma^{g}$. There is a well known identification of $\mathcal{M}\left(\Sigma^{g}\right)$ with the space $\operatorname{Hom}\left(\pi_{1}\left(\Sigma^{g}\right), S U(2)\right) / S U(2)$ of conjugacy classes of representations of the fundamental group $\pi_{1}\left(\Sigma^{g}\right)$ into $S U(2)$. Additionally, this space admits a symplectic form $\Omega$ (see [1] for the details.)

Now, given any simple closed loop $C$ on $\Sigma^{g}$, we obtain a function $f_{C}$ on the space $\operatorname{Hom}\left(\pi_{1}\left(\Sigma^{g}\right), S U(2)\right) / S U(2)$, sending a representation class $\rho$ to

$$
f_{C}(\rho)=\frac{1}{\pi} \arccos \left(\frac{1}{2} \operatorname{tr}(\rho([C]))\right) \in[0,1]
$$

where $[C]$ is the class of $C$ in $\pi_{1}\left(\Sigma^{g}\right)$. In [4] Jeffrey and Weitsman proved that $f_{C}$ is a Hamiltonian function for a $U(1)$-action on a large open dense subset, $U_{C}=f_{C}^{-1}((0,1))$, of $\operatorname{Hom}\left(\pi_{1}\left(\Sigma^{g}\right), S U(2)\right) / S U(2)$. Moreover, if $C$ and $C^{\prime}$ are two simple closed loops in $\Sigma^{g}$ with $[C] \neq\left[C^{\prime}\right]$, then the functions $f_{C}$ and $f_{C^{\prime}}$ commute $\left(\left\{f_{C}, f_{C^{\prime}}\right\}_{\Omega}=0\right)$ and the Hamiltonian 
flows of $f_{C}$ and $f_{C^{\prime}}$ induce an action of a 2-torus, $U(1) \times U(1)$, on $U_{C} \cap U_{C^{\prime}} \subseteq M\left(\Sigma^{g}\right)$. If a third loop $C^{\prime \prime}$ exists, homotopy inequivalent to $C$ and $C^{\prime}$, then we obtain a 3 -torus action, and we may continue this process provided that additional curves can be found.

\subsection{The Case of a Trinion}

As an example, let $D$ be a trinion (a 2 -sphere with three disjoint discs deleted) and let $C_{1}$, $C_{2}$, and $C_{3}$ be the three boundary circles of $D$. Denote by $\mathcal{M}(D)$ the moduli space of gauge equivalence classes of flat $S U(2)$ connections on $D$.

In [4] it was shown that the map

$$
f=\left(f_{C_{1}}, f_{C_{2}}, f_{C_{3}}\right): \mathcal{M}(D) \rightarrow \mathbb{R}^{3}
$$

sends $\mathcal{M}(D)$ bijectively to the set of triples $\left(x_{1}, x_{2}, x_{3}\right)$ satisfying the inequalities

$$
\begin{array}{r}
x_{1}+x_{2}+x_{3} \leq 2 \\
x_{1}+x_{2}-x_{3} \geq 0 \\
x_{1}-x_{2}+x_{3} \geq 0 \\
-x_{1}+x_{2}+x_{3} \geq 0 .
\end{array}
$$

In other words, the image of $f$ is the tetrahedron in $[0,1]^{3}$ whose vertices are $(0,0,0),(1,1,0)$, $(1,0,1)$, and $(0,1,1)$. We will hereafter denote this tetrahedron by $P(D)$.

\subsection{Toric Structures Coming From Trinion Decompositions}

On $\Sigma^{g}$, a maximal collection of pairwise disjoint, non-homotopic, simple closed loops has size $3 g-3$. Let $\mathcal{C}=\left\{C_{1}, C_{2}, \ldots, C_{3 g-3}\right\}$ be such a collection. Such a set is called a marking of the Riemann surface $\Sigma^{g}$.

Recall that a trinion decomposition of $\Sigma^{g}$ is a realization of the surface as a union of $2 g-2$ trinions $\mathcal{D}=\left\{D_{1}, D_{2}, \ldots, D_{2 g-2}\right\}$ glued together along their boundary circles. Given such a decomposition, we obtain a marking of $\Sigma^{g}$ by taking our set $\mathcal{C}$ to be the collection of the $3 g-3$ common boundary circles along which the various trinions in $\mathcal{D}$ are joined. On the other hand, it is easy to see that any marking of $\Sigma^{g}$ gives rise to a trinion decomposition of the surface. 
Let us suppose that we are given a particular trinion decomposition of $\Sigma^{g}$. Let $\mathcal{D}=$ $\left\{D_{1}, D_{2}, \ldots, D_{2 g-2}\right\}$ be the set of trinions in the decomposition, and let $\mathcal{C}=\left\{C_{1}, C_{2}, \ldots, C_{3 g-3}\right\}$ be the corresponding marking of $\Sigma^{g}$. For each curve $C_{i} \in \mathcal{C}$ we have the function $f_{i}=f_{C_{i}}$ (cf. equation (1)) and the set $U_{i}=f_{i}^{-1}((0,1))$. Let $U=\bigcap_{i=1}^{3 g-3} U_{i}$.

We now state, without proof, two key facts from [4] and [5].

Proposition 1 The marking $\mathcal{C}$ determines a $3 g-3$ dimensional torus $K=\mathbb{R}^{3 g-3} / \Lambda$ which acts effectively on $U$, preserving the symplectic form. The lattice $\Lambda$ has rank $3 g-3$ and is spanned by the $3 g-3$ standard basis vectors $e_{i}$ in $\mathbb{R}^{3 g-3}$, along with the vectors $g_{j}=$ $\frac{1}{2}\left(e_{j_{1}}+e_{j_{2}}+e_{j_{3}}\right)$ (for $j=1,2, \ldots, 2 g-2$,) where $C_{j_{1}}, C_{j_{2}}$, and $C_{j_{3}}$ are the three boundary circles of the trinion $D_{j} \in \mathcal{D}$.

Proposition 2 Let $f=\left(f_{1}, f_{2}, \ldots, f_{3 g-3}\right): \mathcal{M}\left(\Sigma^{g}\right) \rightarrow \mathbb{R}^{3 g-3}$. The restriction of $f$ to the set $U$ is the moment map for the action of the torus $K$, and the closure of the image of this moment map is a convex polyhedron $P$ of dimension $3 g-3$. Let $D_{j} \in \mathcal{D}$ with boundary circles $C_{j_{1}}, C_{j_{2}}$, and $C_{j_{3}}$, and denote by $\pi_{j}$ the projection $\mathbb{R}^{3 g-3} \rightarrow \mathbb{R}^{3}$ defined by $\left(x_{1}, x_{2}, \ldots, x_{3 g-3}\right) \mapsto$ $\left(x_{j_{1}}, x_{j_{2}}, x_{j_{3}}\right)$. For every $j \in\{1,2, \ldots, 2 g-2\}$ the image of the composition $\pi_{j} \circ f$ is the tetrahedron $P(D)$. The polytope $P$ is the intersection $\bigcap_{j=1}^{2 g-2} \pi_{j}^{-1}(P(D))$.

Alternatively, the polytope $P$ can be described as the set of all points $\left(x_{1}, x_{2}, \ldots, x_{3 g-3}\right) \in$ $\mathbb{R}^{3 g-3}$ such that for each $j \in\{1,2, \ldots, 2 g-2\}$ the triple $\left(x_{j_{1}}, x_{j_{2}}, x_{j_{3}}\right)$ satisfies the inequalities of the previous section, where again, the indices $j_{1}, j_{2}$, and $j_{3}$ correspond to the three boundary circles of the $j$ th trinion in $\mathcal{D}$. In particular, $P$ is always contained inside the unit cube $[0,1]^{3 g-3}$.

\section{From Trivalent Graphs to Toric Varieties}

Let $\Gamma$ be a trivalent graph of genus $g$. Note that we will allow for the possibility that $\Gamma$ has loops (edges connecting a vertex to itself) or multi-edges (two vertices in $\Gamma$ may be connected with more than one edge.) Let $V(\Gamma)$ and $E(\Gamma)$ denote respectively the vertices and edges of $\Gamma$. Counting loops as two edges, we have $|E(\Gamma)|=3 g-3$, and $|V(\Gamma)|=2 g-2$.

Such a graph gives us a genus $g 2$-manifold $\Sigma^{g}$ equipped with a marking (or trinion decomposition) in the following way (see [6]): pump up the vertices and edges of $\Gamma$ to 2 spheres and tubes respectively. The result is the manifold $\Sigma^{g}$, and homotopy classes of meridian circles of each of the tubes in the pumped up graph define a set $\mathcal{C}$ of $3 g-3$ disjoint, homotopy inequivalent, simple closed loops on the surface.

Applying propositions 1 and 2 from the previous section, we obtain from the graph $\Gamma$ a convex polytope $P(\Gamma) \in \mathbb{R}^{3 g-3}$, and a lattice $\Lambda(\Gamma)$ for the action of the $3 g-3$ dimensional 
torus $K(\Gamma)$. This information is, in turn, all that is required to completely determine a toric variety, the toric variety associated to the graph $\Gamma$, which we will denote by $\mathcal{V}(\Gamma)$.

The toric varieties corresponding to trinion decomposed surfaces were introduced by Jeffrey and Hurtubise in [3]. A primary focus in [6] is a certain class of trivalent graphs, the so-called multi-theta graphs, and their corresponding toric varieties. It is to this case that we now turn our attention.

\subsection{Multi-Theta Graphs}

The multi-theta graph of genus $g$, denoted $\Theta_{g}$, is best described (as in [6]) as a vertical oval $O$ crossed by $g-1$ horizontal edges. The $2 g-2$ vertices of the graph are separated by a vertical axis of symmetry into two groups of size $g-1$. Each vertex is joined by an edge to the vertices immediately above and below, and its "twin" opposite the axis of symmetry (with the obvious exception of the top pair and the bottom pair, which are connected to each other by a double edge).

Example: $\mathbf{g}=\mathbf{2}$. In this case, our (multi-)theta graph consists of two vertices joined by three edges. The marking for the corresponding surface $\Sigma^{2}$ consists of three curves $\mathcal{C}=\left\{C_{1}, C_{2}, C_{3}\right\}$, each curve coming from some edge in the graph. The underlying trinion decomposition for $\Sigma^{2}$ consists of two trinions $D_{1}$ and $D_{2}$ glued together along their three boundary circles. We see that, according to proposition 2, the three dimensional polytope $P\left(\Theta_{2}\right)$ is none other than the tetrahedron $P(D)$ from the previous section.

Now, the lattice $\Lambda\left(\Theta_{2}\right)$ is spanned by $e_{1}=(1,0,0), e_{2}=(0,1,0)$, and $e_{3}=(0,0,1)$, together with $g_{1}=g_{2}=\left(\frac{1}{2}, \frac{1}{2}, \frac{1}{2}\right)$. Let $v_{i}=g_{1}-e_{i}$, for $i \in\{1,2,3\}$, so that $\Lambda\left(\Theta_{2}\right) \cong$ $\mathbb{Z} v_{1} \oplus \mathbb{Z} v_{2} \oplus \mathbb{Z} v_{3}$. Evidently, $P\left(\Theta_{2}\right)$ is a lattice polytope with respect to $\Lambda\left(\Theta_{2}\right)$. One can verify that the normal fan of $P\left(\Theta_{2}\right)$ - the fan generated by the inward-pointing normals to the facets of $P\left(\Theta_{2}\right)$ - is a strongly convex complete simplicial fan. More is true, for we may define an isomorphism of the lattice $\Lambda\left(\Theta_{2}\right)$ with the standard lattice $\mathbb{Z}^{3}$ using

$$
A=\left[\begin{array}{lll}
0 & 1 & 1 \\
1 & 0 & 1 \\
1 & 1 & 0
\end{array}\right],
$$

and additionally, $A$ maps the normal fan of $P\left(\Theta_{2}\right)$ to the fan generated by $(1,0,0),(0,1,0)$, $(0,0,1)$, and $(-1,-1,-1)$. This is the normal fan for the standard 3-simplex $\Delta^{3}$. It follows that the toric variety for the pair $\left(P\left(\Theta_{2}\right), \Lambda\left(\Theta_{2}\right)\right)$ is the same as the variety for the pair $\left(\Delta^{3}, \mathbb{Z}^{3}\right)$, which is known to be $\mathbb{C} P^{3}$. 


\section{2 $P\left(\Theta_{g}\right)$ and the Variety $\mathcal{V}\left(\Theta_{g}\right)$}

We conclude with two straightforward facts about the polytope $P\left(\Theta_{g}\right)$.

Lemma 1 Exactly $2^{g}$ of the vertices of $P\left(\Theta_{g}\right)$ are vertices of the unit cube $[0,1]^{3 g-3}$.

Proof. Since $P\left(\Theta_{g}\right)$ is contained within $[0,1]^{3 g-3}$, if $x \in P\left(\Theta_{g}\right)$ and $x$ is itself a vertex of $[0,1]^{3 g-3}$, then $x$ is necessarily a vertex of $P\left(\Theta_{g}\right)$. Now, if $x$ is a vertex of the unit cube, then $x \in P\left(\Theta_{g}\right)$ if its image under each of $2 g-2$ projections $\pi_{j}$ is a vertex of the tetrahedron $P(D)$ (cf. proposition 2.) Such points correspond to labellings of the edges of $\Theta_{g}$ with either a 0 or a 1 , such that for each vertex $v \in V\left(\Theta_{g}\right)$, the triple of edges at $v$ are either all labelled 0 , or exactly one is labelled 0 .

Beginning with the top pair of vertices in $\Theta_{g}$, we see that there are exactly four admissible ways to label the group of edges emanating from the pair. After a choice has been made for the top pair, there are two possible labellings for the undetermined edges adjacent to the next pair. And, for each of the remaining $g-3$ pairs of vertices there are always two possible labellings, regardless of how the previous pair's edges were labelled. This gives a total of $4\left(2^{g-2}\right)=2^{g}$ possible labellings.

Remark. It must be noted that the method for counting vertices of $P\left(\Theta_{g}\right)$ in the above argument is not exhaustive for $g \geq 3$. That is, requiring that $\pi_{j}(x)$ be a vertex of $P(D)$ for every $j \in\{1,2, \ldots, 2 g-2\}$ is enough to determine that $x$ is a vertex of $P\left(\Theta_{g}\right)$, but not necessary.

Proposition 5.4 of [6] asserts that all of the vertices of the polytope $P\left(\Theta_{g}\right)$ are vertices of $[0,1]^{3 g-3}$, that there are $2^{g}$ in total, and that they are of the form $\left(\star, \star^{\prime}, 0, \star, \star^{\prime}, 0, \star, \ldots, \star\right)$, or $\left(\star, \star^{\prime}, 1, \star, \star^{\prime}, 1, \star, \ldots, \star\right)$, where $\star$ and $\star^{\prime}$ are chosen freely from $\{0,1\}$. We now see that this cannot be true. For example, the above argument shows us that the point $(1,1, \ldots, 1)$ cannot be a vertex of $P\left(\Theta_{g}\right)$ for any $g$, as is claimed. This can also be seen by noting that $(1,1, \ldots, 1)$ does not satisfy the inequalities of section 1.3 for any trinion in the decomposition of $P\left(\Theta_{g}\right)$.

In the previous section we saw that $\mathcal{V}\left(\Theta_{2}\right) \cong \mathbb{C} P^{3}$. One might ask whether or not any of the other varieties $\mathcal{V}\left(\Theta_{g}\right)$ are also singularity free. Unfortunately, as we shall soon see, this cannot be the case.

Definition 1 An $n$ dimensional polytope $P$ in $\mathbb{R}^{n}$ is said to be simple if its 1 -skeleton is an $n$-regular graph. 
Equivalently, an $n$ dimensional polytope $P$ is simple if exactly $n$ facets of $P$ meet at each vertex. It is a well-known fact that a smooth toric variety must have a simple moment polytope.

Lemma 2 For every $g \geq 3$, the polytope $P\left(\Theta_{g}\right)$ is non-simple.

Proof. It follows from the proof of the previous lemma that the origin is always a vertex of $P\left(\Theta_{g}\right)$. Now, from each trinion $D_{j}$ in the decomposition of the underlying surface $\Sigma^{g}$ we are given the set of four inequalities:

$$
\begin{aligned}
x_{j_{1}}+x_{j_{2}}+x_{j_{3}} & \leq 2 \\
x_{j_{1}}+x_{j_{2}}-x_{j_{3}} & \geq 0 \\
x_{j_{1}}-x_{j_{2}}+x_{j_{3}} & \geq 0 \\
-x_{j_{1}}+x_{j_{2}}+x_{j_{3}} & \geq 0 .
\end{aligned}
$$

As we have seen, the $2 g-2$ sets of inequalities of the above type define the polytope $P\left(\Theta_{g}\right)$. Consider the last three inequalities in the above set. Each defines an affine half-space in $\mathbb{R}^{3 g-3}$, and each of these half-spaces supports a different facet of $P\left(\Theta_{g}\right)$ containing the origin. There are $3(2 g-2)=6 g-6$ such facets, and so $P\left(\Theta_{g}\right)$, which has dimension $3 g-3$, cannot be simple.

Remark. It follows from this that the variety $\mathcal{V}\left(\Theta_{g}\right)$ is singular for $g \geq 3$. Moreover, the above argument applies to any polytope $P(\Gamma)$, so long as $\Gamma$ is loop-free (note that the origin is, in fact, always a vertex of $P(\Gamma)$.) So the variety $\mathcal{V}(\Gamma)$ is singular whenever $\Gamma$ is a loop-free trivalent graph of genus $g \geq 3$. In proposition 5.5 of [6] it was asserted that not only is the polytope $P\left(\Theta_{g}\right)$ simple, but also that the set of edges emanating from any vertex of $P\left(\Theta_{g}\right)$ forms a rational basis for $\mathbb{R}^{3 g-3}$. In other words, it was claimed that the polytope $P\left(\Theta_{g}\right)$ is Delzant, and consequently that the corresponding toric variety $\mathcal{V}\left(\Theta_{g}\right)$ - written there as $D M_{g}$ - is always smooth. This is plainly false, since $P\left(\Theta_{g}\right)$ is not even simple.

\section{References}

[1] W. M. Goldman, The Symplectic Nature of Fundamental Groups of Surfaces. Adv. in Math. 54 (1984), no. 2, 200-225.

[2] W. M. Goldman, Invariant Functions on Lie Groups and Hamiltonian Flows of Surface Group Representations. Invent. Math. 85 (1986), no. 2, 263-302. 
[3] J. Hurtubise and L. C. Jeffrey, Representations with Weighted Frames and Framed Parabolic Bundles. Canad. J. Math. 52 (2000), no. 6, 1235-1268.

[4] L. C. Jeffrey and J. Weitsman, Bohr-Sommerfeld Orbits in the Moduli Space of Flat Connections and the Verlinde Dimension Formula. Commun. Math. Phys. 150 (1993), 593-630.

[5] L. C. Jeffrey and J. Weitsman, Toric Structures on the Moduli Space of Flat Connections on a Riemann Surface: Volumes and the Moment Map. Adv. in Math. 106 (1994), 151-168.

[6] A. Tyurin, Delzant Models of Moduli Spaces. Investiya RAN: Ser. Mat. 67:2 (2003), $167-180$.

James J. Uren, Department of Mathematics, University of Toronto, Toronto, Ontario M5S 3G3, Canada

email address: jjuren@math.toronto.edu 